\title{
Inconsistent stability of Newmark's method in structural dynamics applications
}

\author{
Richard Wiebe \\ University of Washington \\ 201 More Hall, Box 352700 \\ Seattle, WA 98195-2700 \\ Email: rwiebe@co.uw.edu \\ Ilinca Stanciulescu * \\ Rice University \\ 208 Ryon Laboratory, MS 318 \\ 6100 Main St, Houston, TX 77005 \\ Email: ilinca.s@rice.edu
}

\begin{abstract}
The stability of numerical time integrators, and of the physical systems to which they are applied, are normally studied independently. This conceals a very interesting phenomenon, here termed inconsistent stability, wherein a numerical time marching scheme predicts a stable response about an equilibrium configuration that is, in fact, unstable. In this paper, time integrator parameters leading to possible inconsistent stability are first found analytically for conservative systems (symmetric tangent stiffness matrices), then several structural arches with increasing complexity are used as numerical case studies. The intention of this work is to highlight the potential for this unexpected, and mostly unknown, behavior to researchers studying complex dynamical systems, especially through time marching of finite element models. To allow for direct interpretation of our results, the work is focused on the Newmark time integrator, which is commonly used in structural dynamics.
\end{abstract}

${ }^{*}$ Corresponding author 


\section{Introduction}

Time integration schemes are evaluated by two criteria: stability, and accuracy. A stable time integrator is one which will produce a solution that remains bounded, while an unstable one will 'blow up' as the error accumulates ever more rapidly. Of course, real systems do not diverge to infinity, so instability of time integrators is also a sign of inaccuracy, however, stability does not necessarily imply that the prediction is close to the 'true' solution, only that it remains bounded. The stability of time integrators is frequently studied in a linear context [1]. This is out of necessity, as nonlinearities usually make finding analytical stability bounds intractable. Nonetheless, the linear stability bounds provide excellent guidelines for nonlinear systems, and are even exact in a local sense under linearization of the equation of motion. In many cases nonlinear systems do not, however, diverge to infinity as the higher-order terms may keep the output of the integrator bounded. Accuracy is therefore perhaps of more concern in nonlinear systems than in linear systems, as instabilities may not be obvious when inaccuracies pollute the solution.

The question of accuracy contains many subtleties, particularly for nonlinear systems. For instance, in a system such as the harmonically-forced double-well Duffing equation, a time integrator may yield a trajectory (given some initial conditions) that traces out a path that ends up settling into a single-well response in the left potential well, while the exact solution settles in the right potential well. Similarly, chaotic trajectories will also inevitably lead to quantitative differences under different time steps and integration schemes [2]. Of course, the exact solution is never obtainable, outside of some exactly integrable systems [3], which by the fact that they follow a closed-form solution cannot exhibit complex types of behavior such as chaos. Thus, when two integrators, or an integrator with two different time steps, yield different solutions, there is no 'truth' model to appeal to. In practice, this is not as critical as it may seem; one must simply include some investigation into the robustness of competing solutions, and be willing to lose the notion that some of these solutions are 'right' and others are 'wrong', when they merely point out the sensitivity of the system.

The stability of time integrators can be contrasted with the stability of real systems. The static stability of conservative structures has been discussed thoroughly in [4], among others. The stability of static equilibria may be interrogated by observing whether the dynamic response in their vicinity diverges, or remains bounded, indicating unstable and stable equilibria respectively. There are four possible scenarios in modeling the static and dynamic response of structures in the vicinity of equilibria; the solution and real response are i) stable-stable, ii) unstable-unstable, iii) unstable-stable, iv) stable-unstable.

Only the latter two cases are problematic. The third case, in which the integrator diverges erroneously, has traditionally been the prime concern in the development of time integrators. For example, in [5] an approximate condition for sufficient stability of the Newmark time integrator in nonlinear systems is found. We instead consider the fourth scenario, in which 
the real response is divergent, and yet the integrator produces a stable oscillation. From an engineering perspective, case iv) produces an under-conservative prediction of the response and may therefore be more critical than case iii).

\section{Theory}

Nonlinearities are present in virtually all engineering systems while only few are simple enough to allow access to analytical solutions. Therefore, for an accurate representation of the solution of these systems, robust numerical algorithms are necessary, particularly ones that are capable of handling the specific nonlinearities that are analyzed.

The results in this paper are partially based on an earlier publication on the phenomenon of inconsistent stability under co-simulation using fourth-order Runge-Kutta (RK4) [6]. In this paper we instead focus on the Newmark method [7], as it is the method of choice in the field of structural mechanics for time integration. We also test the theoretical results using multiple numerical case studies, which was not the focus of [6].

\subsection{Physical and Numerical Stability}

In this context, of great interest is the examination of the relationship between the stability of the physical system (or of its continuum mathematical model) and the stability of its discretized representation. When analyzing the performance of a time integrator it is quite common to refer to unconditional stability as one of the most desirable properties that such an algorithm should exhibit. Such property guarantees that numerically obtained solutions are stable (e.g., remain bounded). However, complex dynamical systems often have unstable solutions that are physical (true representation of the system response). For such systems, instead of unconditionally stable integrators we need to seek algorithms with consistent stability.

Consider for instance the equation of motion of a single degree of freedom (SDOF) system in the absence of external forcing: $m \ddot{x}+c \dot{x}+k x=0$. Using the notation $y=\dot{x}$ it can be expressed as a first order two dimensional system $(\dot{x}, \dot{y})^{T}=$ $\mathbf{A}(x, y)^{T}$, whose equilibrium solution $(x=0, y=0)$ is asymptotically stable if and only if $\Gamma=\operatorname{Tr}(\mathbf{A})<0$ and $\Delta=\operatorname{Det}(\mathbf{A})>0$. The zero solution is marginally stable if $(\Gamma, \Delta)$ belongs to the stability boundary $\{(\Gamma, \Delta) \mid \Gamma=0, \Delta \geq 0$ or $\Gamma \leq 0, \Delta=0\}$. The region defined by $\Gamma \leq 0, \Delta \geq 0$ is the stability region of the continuous system (Fig.1a). Application of a time integration algorithm is then expressed as a two-dimensional discrete map $\left(d_{n+1}, v_{n+1}\right)^{T}=\mathbf{D}\left(d_{n}, v_{n}\right)^{T}$ whose solution is asymptotically stable if and only if

$$
|\operatorname{Tr}(\mathbf{D})|-1<\operatorname{Det}(\mathbf{D})<1
$$

and marginally stable if $\operatorname{Det}(\mathbf{D})=1 \operatorname{or} \operatorname{Det}(\mathbf{D})=|\operatorname{Tr}(\mathbf{D})|-1$ ( [8], p. 153). The stability region for the discrete system is 


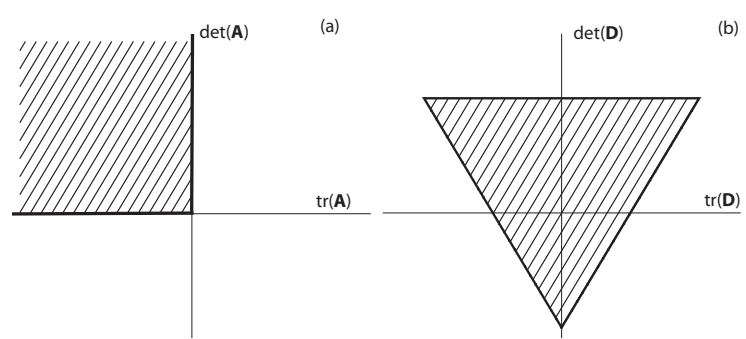

Fig. 1: Stability regions of the continuous (a) and discrete (b) systems

shown in Fig. 1b. The stable integrator - unstable system scenario is avoided when the projection of the stability region of 1(b) onto the axes of the continuous system is fully inside the stability region of 1(a). For an extended discussion the interested reader is referred to [6].

\subsection{The Newmark Method}

The equation of motion for the undamped free vibrations of a single degree of freedom system is $m \ddot{x}+k x=0$, where $m \geq 0$ is the mass and $k$ the stiffness. As a first order system, it is written in matrix form as

$$
\left(\begin{array}{c}
\dot{x} \\
\dot{y}
\end{array}\right)=\left(\begin{array}{cc}
0 & 1 \\
-\omega_{0}^{2} & 0
\end{array}\right)\left(\begin{array}{l}
x \\
y
\end{array}\right),
$$

where $y=\dot{x}$, and $\omega_{0}^{2}=k / m$. We will use this system to discuss some properties of the Newmark class of time integrators. The system is stable for $\omega_{0}^{2} \geq 0$. The discretized form using Newmark's integrator ( [1] p. 490) is based on enforcing the equilibrium at step $\mathrm{n}+1, a_{n+1}+\omega_{0}^{2} d_{n+1}=0$, together with the updates:

$$
\begin{aligned}
& d_{n+1}=d_{n}+\Delta t v_{n}+\frac{\Delta t^{2}}{2}\left[(1-2 \beta) a_{n}+2 \beta a_{n+1}\right] \\
& v_{n+1}=v_{n}+\Delta t\left[(1-\gamma) a_{n}+\gamma a_{n+1}\right]
\end{aligned}
$$

$d_{n}, v_{n}$, and $a_{n}$ are the discretized representations of the displacement $x$, velocity $\dot{x}$ and acceleration $\ddot{x}$ at step $n, \Delta t$ is the time step (considered constant for this analysis) and $\beta$ and $\gamma$ are algorithmic parameters. The trace and determinant of the discrete operator are obtained as

$$
\begin{aligned}
\operatorname{Tr}(\mathbf{D}) & =\frac{4-\omega_{0}^{2} \Delta t^{2}(1-4 \beta+2 \gamma)}{2+2 \omega_{0}^{2} \Delta t^{2} \beta} \\
\operatorname{Det}(\mathbf{D}) & =\frac{2+\omega_{0}^{2} \Delta t^{2}(1+2 \beta-2 \gamma)}{2+2 \omega_{0}^{2} \Delta t^{2} \beta}
\end{aligned}
$$

Unconditional stability is obtained for $2 \beta \geq \gamma \geq \frac{1}{2}$, which represents the intersection of the domains defined by Eq. (1) for all positive $\omega_{0}^{2}$. Inconsistent stability occurs when Eq. (1) is satisfied, despite the system being physically unstable. An example 

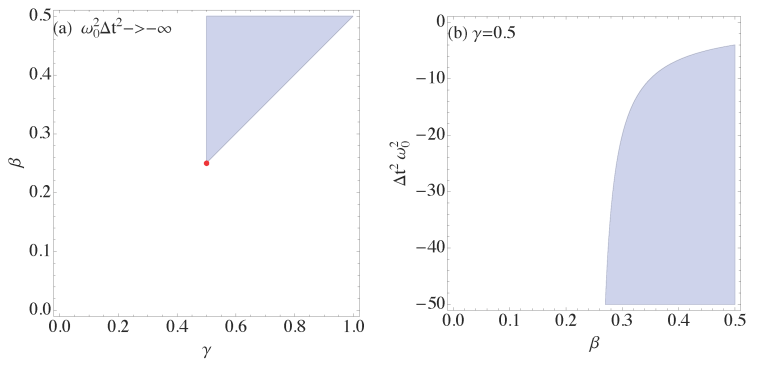

Fig. 2: Stability domain of discrete solution for unstable physical systems (a) $\omega_{0}^{2} \Delta t^{2} \rightarrow-\infty$; (b) $\gamma=0.5$

of this is shown in Fig. 2, where for an unstable system $\left(\omega_{0}^{2}<0\right)$, a combination of algorithmic parameters from the shaded region will predict stable responses. Part (a) shows the unconditional stability domain in the limit $\omega_{0}^{2} \Delta t^{2} \rightarrow-\infty$. A member of the Newmark family of methods that is often used is the trapezoidal rule $\left(\gamma=\frac{1}{2}, \beta=\frac{1}{4}\right.$, represented as a dot in Fig. 2a).

It is easy to show that for a physically unstable system $\left(\omega_{0}^{2}<0\right)$, where the denominator in the expression for the determinant is negative, i.e., $2+2 \beta \omega_{0}^{2} \Delta t^{2}<0$, the determinant inequality in Eq. (1) yields $\gamma>1 / 2$. This implies that for $\gamma>1 / 2$ and an unstable system $\left(\omega_{0}^{2}<0\right)$, we must have

$$
\Delta t^{2}>-\frac{1}{\omega_{0}^{2} \beta}
$$

for the system to satisfy the determinant inequality, which is necessary to yield a numerically (erroneously) stable response. This is therefore a necessary condition for inconsistent stability (for $\gamma>1 / 2$ ). This is, however, not a sufficient condition, nor does it imply with any certainty what occurs when any of the inequalities involved are reversed. However, the necessary condition in Eq. (7) with the inequality direction reversed provides a useful starting estimate for the time step in the Newmark method, as it is always of interest to avoid the possibility of inconsistent stability. Given the prevalence of the trapezoidal parameters $\left(\gamma=\frac{1}{2}, \beta=\frac{1}{4}\right)$, the approximate time step check $\Delta t<\sqrt{-2 / \omega_{0}^{2}}$, using the most negative $\omega_{0}^{2}$ value over all of the static equilibria, might prove useful in practice.

The standard trapezoidal Newmark parameters may yield marginal stability in what should be an unstable response. These results are only valid for a linear response, or for the small linearized response about equilibria in nonlinear systems. However, 'perturbations' due to nonlinearities or physical damping may magnify or reduce the likelihood of inconsistent stability and potentially push the standard parameters into the inconsistent stability region.

The results discussed in this section are all for an SDOF system. The extension to higher-order systems is trivial, as long as they can be de-coupled. This is always the case for the linearized dynamics of conservative systems with Rayleigh damping. This simplification is not especially limiting, as many engineering systems have an underlying energy potential, and Rayleigh damping is commonly used in modeling. In principle, the conditions in Eq. (1) could be applied to higher- 
dimensional systems that cannot be de-coupled, which would extend our results beyond conservative systems. However, this is an excessively bulky exercise, so we have limited ourselves to conservative systems.

\section{Case Studies}

In this section we show multiple numerical case studies of systems that may exhibit inconsistent stability. We start with an SDOF system, for which the results above are directly applicable, then follow with 2DOF and MDOF cases in which we exploit de-coupling to examine inconsistent stability. The case studies are all based on the structural arch, which is a classical problem in structural engineering due to (1) the potential existence of multiple stable and unstable static equilibria and snap-through, and (2) nonlinear dynamical behavior similar to that described by the Duffing equation (see [9] for a summary of studies of the Duffing equation).

\subsection{SDOF Arch}

Fig. 3a shows a SDOF arch under a conservative point load. The potential and kinetic energy for this system are given by

$$
\begin{gathered}
V=2 k L^{2}\left(\cos \theta_{0}-\cos \theta\right)^{2}-F L\left(\theta_{0}-\theta\right), \\
T=m L^{2} \dot{\theta}^{2} / 2
\end{gathered}
$$

where the system states are $\theta$ (position), and $\dot{\theta}$ (angular velocity). Likewise, the system parameters are the central lumped mass $m$, the link length $L$ (two equal massless links), the spring stiffness $k$, and the initial angle at which the spring is unstretched $\theta_{0}$. Utilizing a Lagrangian approach, these terms may be used to obtain the undamped equation of motion and the equilibrium relationship;

$$
\begin{gathered}
\ddot{\theta}+\frac{4 k}{m}\left(\cos \theta_{0}-\cos \theta\right) \sin \theta+\frac{F}{m L}=0, \\
F\left(\theta ; \theta_{0}\right)=4 k L\left(\cos \theta-\cos \theta_{0}\right) \sin \theta, \\
\omega_{0}^{2}\left(\theta ; \theta_{0}\right)=\frac{4 k}{m}\left(\cos \theta_{0} \cos \theta+\sin ^{2} \theta-\cos ^{2} \theta\right) .
\end{gathered}
$$

Due to the symmetry of the structure, the unloaded stable equilibria are always $\theta= \pm \theta_{0}$, and the unstable equilibria is always located at $\theta=0$. The linearized (squared) natural frequency in Eq. (12) is the derivative of the restoring force term in Eq. (10). 


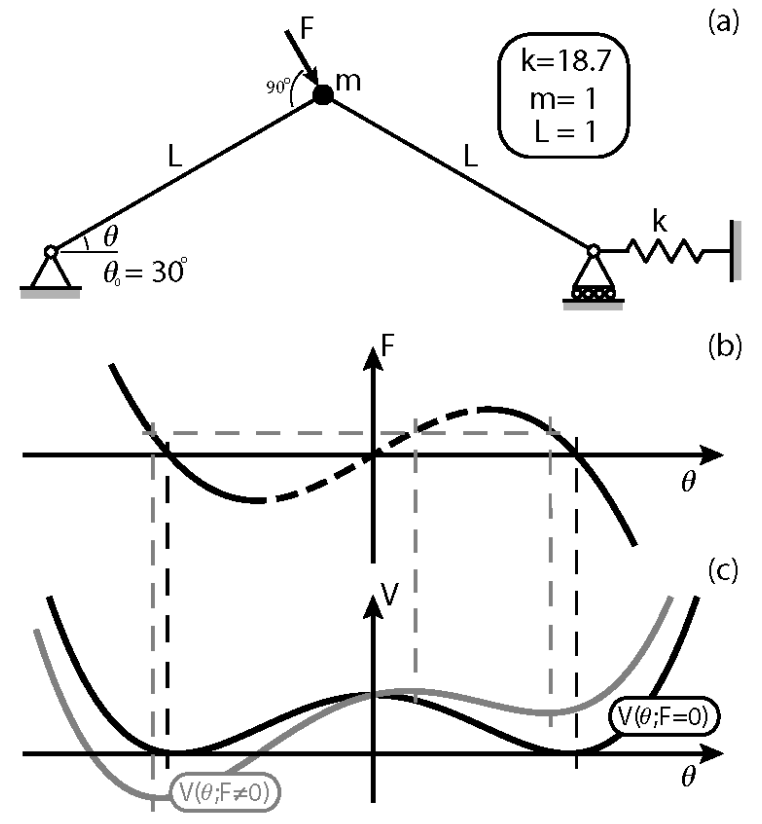

Fig. 3: A single-degree-of-freedom arch that exhibits multiple stable and unstable equilibria. (a) Schematic, (b) static equilibrium relationship, and (c) potential energy for $F=0$, and one example where $F \neq 0$.

Equations (11) and (12) provide the complete picture of the static behavior of the structure as is seen in Fig. 3b, where the dashed region of the equilibrium curve indicates an unstable equilibrium as it has a negative squared linearized natural frequency (i.e., negative stiffness). From the perspective of the potential energy, applying a load shifts the location of the potential energy turning points as is shown by the difference between the gray (loaded) and black (unloaded) potential energy curves in part (c).

In undamped systems, the underlying equilibria are difficult to observe in the dynamic response. The time integrator parameters $(\beta=0.4$ and $\gamma=0.55)$ used herein, however, introduce numerical damping that increases with the size of the time step. Thus, we expect the response to move away from the unstable equilibria. What is not expected, even for very large levels of artificial damping (or real damping), is that the solution would decay to an unstable equilibrium configuration. Fig. 4a shows the unforced response $(F=0)$ determined with the Newmark method discussed in the previous section for various time steps. The three simulations all begin with effectively the same initial conditions $(d t=0.01 \mathrm{~s}$ and $d t=0.10 \mathrm{~s}$ started on opposing sides of symmetric system), however, the largest time step results in a time series that converges to the unstable equilibrium configuration. The smaller time steps result in a more accurate depiction, as they oscillate about, and gradually converge to, the two stable equilibria. Note that substituting Eq. (12) for $F=0$ into Eq. (7) results in $\Delta t>0.50 \mathrm{~s}$ being a necessary condition for inconsistent stability with $\beta=0.4$.

It is important to note that not all initial conditions lead to the inconsistently stable equilibrium for $d t=1.00 \mathrm{~s}$. In Corresponding author: Ilinca Stanciulescu 


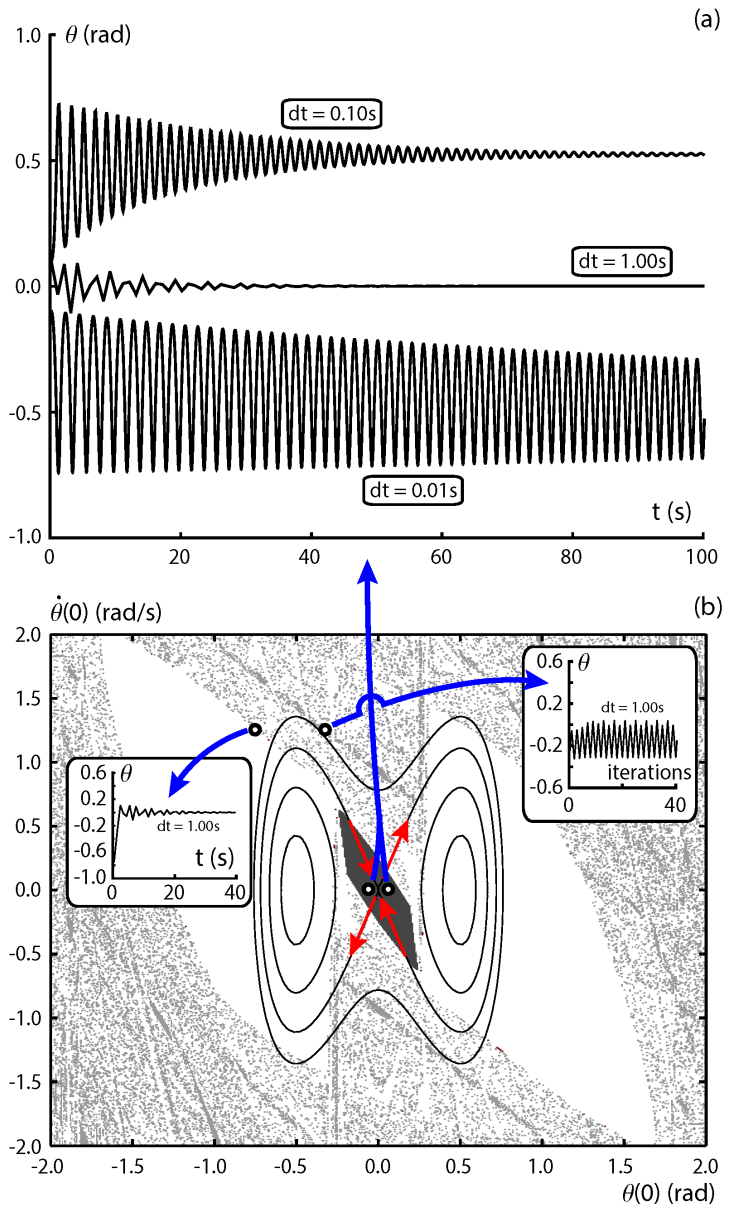

Fig. 4: Unforced response of the SDOF system obtained using the Newmark method $(\beta=0.4$ and $\gamma=0.55)$ for various time steps ( $d t=0.01 \mathrm{~s}$ and $d t=0.10 \mathrm{~s}$ initiated from opposite sides for clarity). (a) Time series showing inconsistent stability for $d t=1.0 \mathrm{~s}$. (b) Basins of attraction for consistent stability (unshaded), inconsistent stability (dark gray), and non-converging (light gray) solutions for $d t=1.0$ s. $F=0.0, x(0)= \pm 0.1, \dot{x}(0)=0.0$.

approximating a smooth two-state system (which has constant energy solutions such as those superimposed in Fig. $4 \mathrm{~b}$ and cannot produce chaos by the Poincare-Bendixon theorem [10]) by a discrete map, one opens the door for many complicated types of responses. Fig. $4 \mathrm{~b}$ shows that, depending on the initial conditions, the system may yield inconsistent stability (dark gray), consistent stability, i.e., attraction to one of the stable equilibria (unshaded), or non-converging solutions that oscillate mostly periodically within the Newmark method iterations (light gray). As might be expected, the dark gray region hovers near the unstable equilibria, and is biased in the direction of the stable manifold (see arrows). The ICs used for part (a) of this figure are shown by the empty circles. Inconsistent stability also occurs farther away in IC space. The boundary that separates the dominant unshaded 'bulbs' from the rest of the plot contains a very thin region of inconsistent stability that does not appear at this resolution. This is seen in the inset on the left side of part (b). A sample non-converging result is also shown in the inset on the right. Instead of plotting time, the horizontal axis shows iterations within a single time step of the 

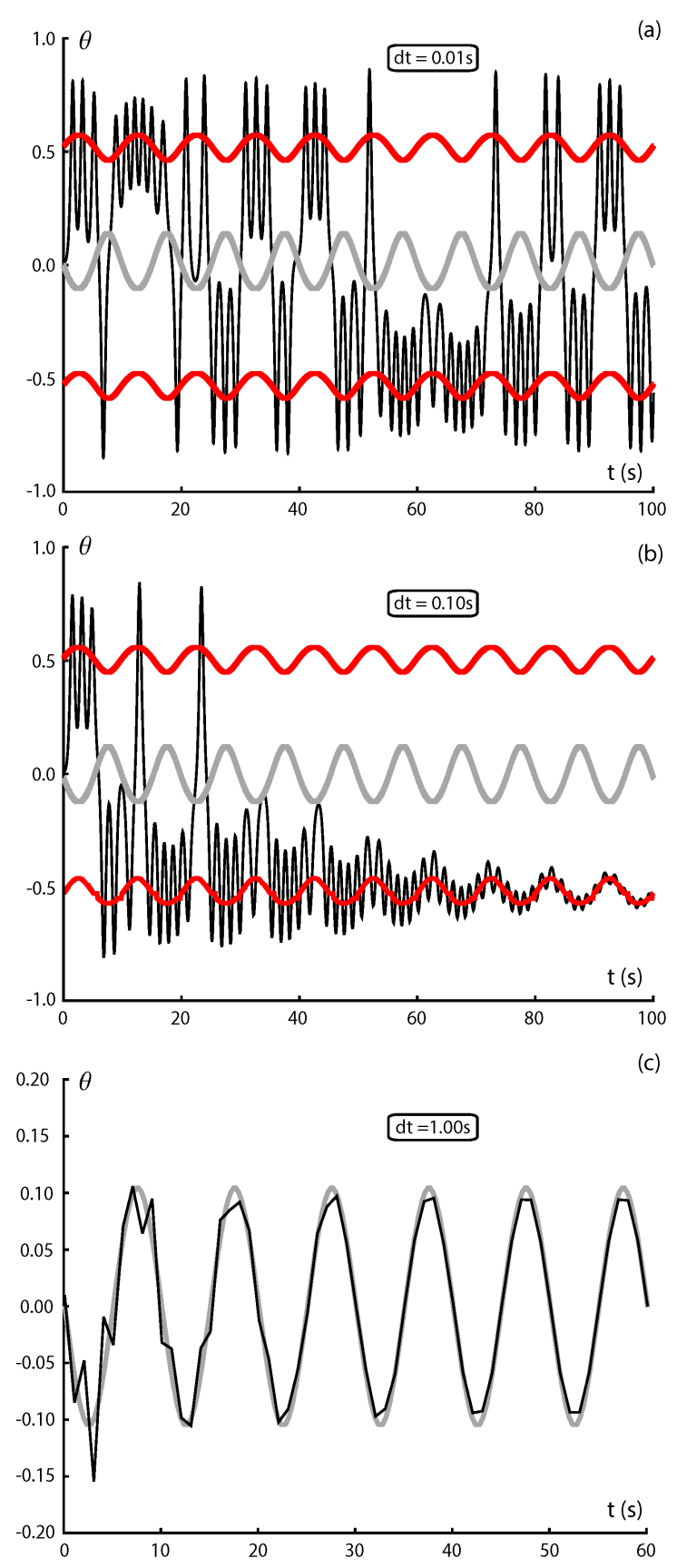

Fig. 5: Forced response of the SDOF system obtained using the Newmark method $(\beta=0.4$ and $\gamma=0.55)$ for various time steps (black) with overlayed instantaneous stable (red/dark shaded curve) and unstable (gray/light shaded curve) static equilibria. $F=0.1 \sin \left(\frac{2 \pi}{10} t\right), x(0)=0.01, \dot{x}(0)=0.0$.

Newmark method.

The time step in which the non-convergence occurs, may not be the first time step. In fact, one could look backward from each time step that leads to a nonconverging result to obtain more ICs in the light gray region. This is true for all types of response, leading to the complex overall picture of interweaving sets that appear to be fractal. The light gray shaded regions are also likely the intersection of many fractals, each for a particular type of non-converging solution, periodic or 
Fig. 6: Unforced response of the SDOF system, now for $k / m=2800$, obtained using the Newmark method with standard parameters $(\beta=0.25$ and $\gamma=0.5)$ for $d t=1 \mathrm{~s}, x(0)=0.1, \dot{x}(0)=0.0$. otherwise.

Fig. 5 shows that inconsistent stability may also occur in the forced response, in this case a harmonic force. The external forcing causes a shift in the equilibrium positions (red/darker-stable, gray/lighter-unstable). While predictions (b) and (c) demonstrate increasing numerical damping, (c) shows a response that is physically impossible as it follows a moving unstable target.

Two useful values in selecting the time step in the simulation of dynamical systems are the natural period of the forcing, and of the linearized structural response. While $d t=1.0 \mathrm{~s}$ may be reasonable when compared to the forcing period $\left(T_{f}=\right.$ $10 \mathrm{~s})$, it is less so in relation to the natural period about the unloaded configuration $\left(T_{0}\left( \pm \theta_{0}\right)=1.5 \mathrm{~s}\right.$ as determined from Eq. (12)). These results suggest that one should also consider the magnitude of the negative stiffness $\omega_{0}^{2}(0)=-10$ when selecting the time step. In this particular example, the negative stiffness of the unstable equilibria is lower (in magnitude) than the positive stiffness of the stable equilibria, and hence a time step chosen based on the stable equilibria would yield good results. Of primary concern are cases where highly unstable equilibria are present in systems with otherwise 'gentle' potential energy surfaces (i.e., low curvature). In this case, time steps that lead to well-behaved (accurate, stable, minimal numerical damping) response in the 'gentle' stable region, may yield inconsistent stability in the region of the unstable equilibria.

A simple inspection of the time series in Fig. 4 shows that the time steps chosen are much too large. Furthermore, the integration parameters used are not typical for the Newmark method. As mentioned earlier, and seen in Fig. 2 (b), the standard parameters are a special case as they tend to marginal stability as $\omega_{0}^{2} \Delta t^{2} \rightarrow-\infty$. Despite this, temporary inconsistent stability is still possible by further increasing the magnitude of the negative stiffness as seen in Fig. 6 for $k / m=2800$. The oscillatory growth may be delayed arbitrarily long by further increasing the magnitude of the negative stiffness, or increasing the time 
step. This occurs because the unstable eigenvalue of the Newmark update approaches -1 from below with increasing $\Delta t^{2} \omega_{0}^{2}$, while the stable eigenvalues shrink to zero (see rapid decay in first time step).

Inconsistent stability is surprising and is an interesting phenomena which should be understood by analysts. Although it is particularly noticeable for the non-standard parameters used in this section, we have also shown that temporary inconsistent stability may occur for the standard Newmark parameters. It is also possible that 'perturbations' from the undamped linearized formulation (e.g., physical damping, nonlinearities) may push the standard parameters inside the inconsistent stability region.

\subsection{Two DOF Arch}
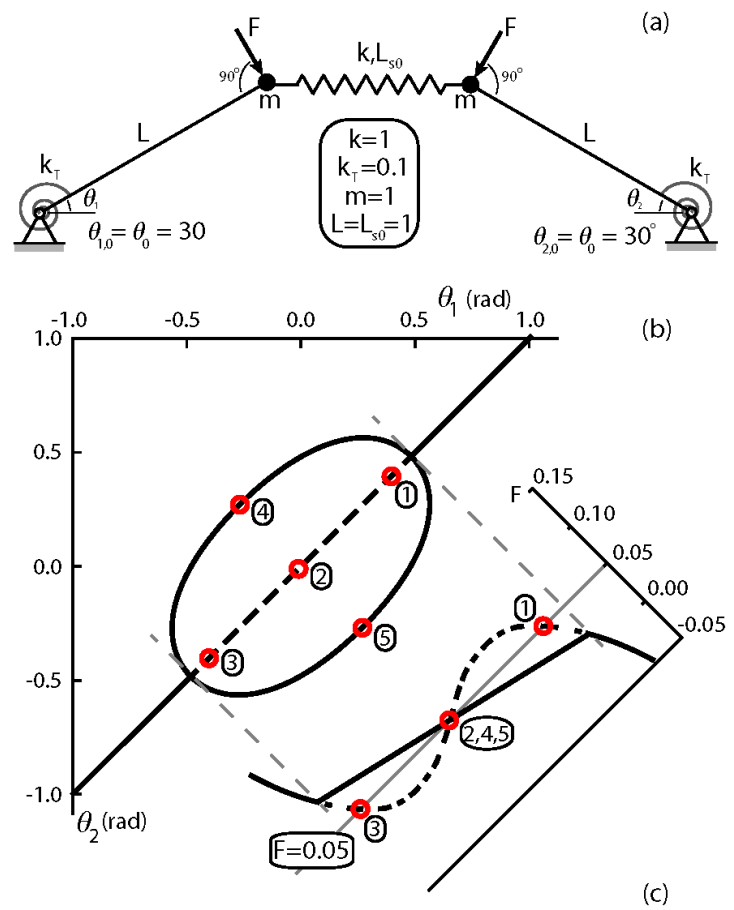

\begin{tabular}{|c|c|c|c|c|c|}
\hline & $\theta_{1 \mathrm{e}}(\mathrm{rad})$ & $\theta_{2 \mathrm{e}}(\mathrm{rad})$ & $\omega_{0.1}^{2}(\mathrm{rad} / \mathrm{s})^{2}$ & $\omega_{02}^{2}(\mathrm{rad} / \mathrm{s})^{2}$ & $\Delta \mathbf{t}_{\mathrm{git}}(\mathrm{s})$ \\
\hline (1) & 0.416 & 0.416 & -0.168 & 0.338 & 3.86 \\
\hline (2) & -0.014 & -0.014 & -0.899 & -0.167 & 1.67 \\
\hline (3) & -0.402 & -0.402 & -0.207 & 0.306 & 3.48 \\
\hline (4) & -0.238 & 0.300 & 0.076 & 1.177 & - \\
\hline (5) & 0.300 & -0.238 & 0.076 & 1.177 & - \\
\hline
\end{tabular}

Fig. 7: A two-degree-of-freedom arch that exhibits multiple stable and unstable equilibria. (a) Schematic, (b) static equilibrium relationship, and (c) stability via natural frequency (squared) of the five equilibria (red empty circles) present under $F=0.05$.

As an intermediate step in complexity, consider the case of a 2DOF arch, as shown in Fig. 7a. The system state is now defined by two angles, and their respective angular velocities. The remaining parameters are labeled in the same manner as 

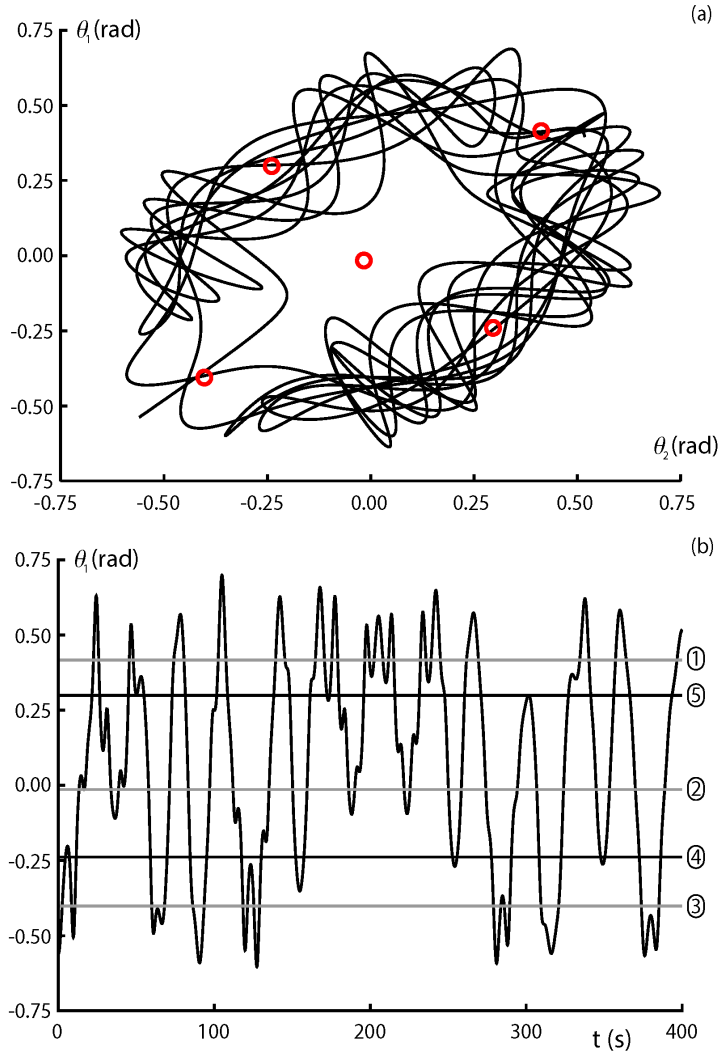

Fig. 8: True response of the 2DOF system under constant force $F=0.05$ obtained using the Newmark method ( $\beta=0.4$ and $\gamma=0.55)$ for $d t=0.1 \mathrm{~s}$. (a) Phase portrait in $\left(\theta_{1}, \theta_{2}\right)$ projection, i.e., angular velocities are not shown. (b) Time series of $\theta_{1}$. The circles denote the positions of the static equilibria.

the SDOF arch, with the addition of the parameter $L_{s 0}$, which is the initial unstretched length of the spring connecting the two identical links. The kinetic and potential energies are given by

$$
\begin{aligned}
V & =V_{s}-F L\left[\left(\theta_{0}-\theta_{1}\right)+\left(\theta_{0}-\theta_{2}\right)\right], \\
V_{s} & =\frac{1}{2} k\left(L_{s}-L_{s 0}\right)^{2}+\frac{1}{2} k_{T}\left[\left(\theta_{0}-\theta_{1}\right)^{2}+\left(\theta_{0}-\theta_{2}\right)^{2}\right], \\
L_{s}^{2} & =L^{2}\left(\sin \theta_{2}-\sin \theta_{1}\right)^{2} \\
& +\left[2 L \cos \theta_{0}+L_{s 0}-L\left(\cos \theta_{1}+\cos \theta_{2}\right)\right]^{2}, \\
T & =\frac{1}{2} m L^{2}\left(\dot{\theta}_{1}^{2}+\dot{\theta}_{2}^{2}\right) .
\end{aligned}
$$

Similarly to the SDOF case, the static equilibria may be obtained by setting the gradient of the potential energy to zero 
$(\boldsymbol{\nabla} V=\mathbf{0})$, and the equation of motion through the Lagrange-Euler equation, which yields

$$
\begin{aligned}
& m L^{2} \ddot{\theta}_{1}+\frac{\partial V}{\partial \theta_{1}}=0, \\
& m L^{2} \ddot{\theta}_{2}+\frac{\partial V}{\partial \theta_{2}}=0 .
\end{aligned}
$$

where $V=V\left(\theta_{1}, \theta_{2} ; \theta_{0}, P\right)$. The linearized equation for small oscillations $u_{1}$ and $u_{2}$ about equilibrium positions $\theta_{1 e}$ and $\theta_{2 e}$ are thus

$$
\begin{aligned}
& m L^{2} \ddot{u}_{1}+\frac{\partial^{2} V}{\partial \theta_{1}^{2}} u_{1}+\frac{\partial^{2} V}{\partial \theta_{1} \partial \theta_{2}} u_{2}=0, \\
& m L^{2} \ddot{u}_{2}+\frac{\partial^{2} V}{\partial \theta_{2} \partial \theta_{1}} u_{1}+\frac{\partial^{2} V}{\partial \theta_{2}^{2}} u_{2}=0 .
\end{aligned}
$$

The expression for the derivatives of $V$ are rather cumbersome, and they have been omitted for brevity. The initial angles in the unloaded configuration are both equal to $\theta_{0}$, which, along with the symmetric loading configuration, makes this a perfectly symmetric structure. Despite this, the structure will deform asymmetrically under load, as the symmetric path in part (b) is unstable while the two mirrored asymmetric equilibrium paths are stable. The dynamic behavior of the structure changes drastically under increasing load levels. The side-on perspective shows that a load of $F=0.05$ leads to a particularly interesting case with 5 equilibria ( 2 stable, 3 unstable). The stability may be determined by reference to the squared natural frequencies of linearized oscillations about the equilibria, where the presence of any negative values indicates instability. These values are displayed in the table in part (c), which shows that equilibria \#1 and \#3 are unstable saddles, and \#2 is an unstable potential hilltop. The final column in this table also shows the critical time step given by the sufficient condition for consistent stability in Eq. (7) based on the most negative (most unstable) squared natural frequency for each of the unstable equilibria.

A sample of the undamped dynamic response of the 2DOF structure is shown in Fig. 8. Part (a) shows the response in a projection of the phase space (angles only) with the five equilibria superimposed. Part (b) likewise, shows a time series of the response of angle $\theta_{1}$ alone. A convergence study, and comparison with RK4, both revealed the time step $d t=0.1 \mathrm{~s}$ used to generate this figure was accurate, and may be seen as the 'true' response. Note that the Newmark parameters used are not the standard ones, however, due to the small time step, the artificial damping is negligible.

Inconsistent stability is demonstrated, once again for larger time steps, in Fig. 9. In part (a), although the response is clearly incorrect (the real system is undamped while the time integrator adds damping), it is at least decaying to a stable solution. The larger time step in part (b), however, leads to inconsistent stability in the vicinity of all three of the unstable 


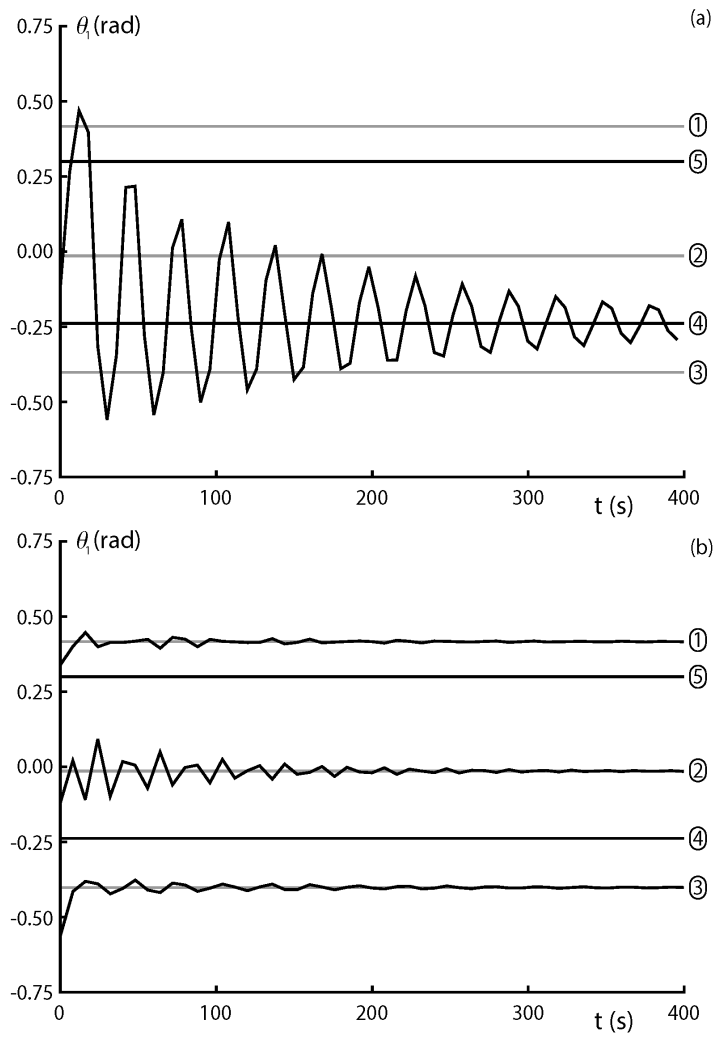

Fig. 9: Consistent and inconsistent stability of the 2DOF system under dead load $F=0.05$ obtained using the Newmark method ( $\beta=0.4$ and $\gamma=0.55$ ) for various time steps. (a) Consistent stability for $d t=6 \mathrm{~s}$. (b) Inconsistent stability for $d t=8 \mathrm{~s}$. All responses initiated near unstable equilibria.

equilibria. The response (shown in $\theta_{1}$ only) does indeed tend to the unstable equilibrium configurations. Note that even the simulated response in part (a), which correctly diverges from the unstable equilibrium (albeit with excessive numerical damping), is for a time step that is well above the necessary condition for inconsistent stability. This emphasizes that Eq. (7) offers a conservative estimate for the size of the time step necessary to avoid inconsistent stability.

The application of Eq. (7), which was based on an SDOF system, to this 2DOF system may seem suspect at first. However, the natural frequencies reported in Fig. 7 are also the natural frequencies of the de-coupled system of SDOF equations in the modal space. The restriction to conservative system ensures that the linearized behavior near an equilibrium will always be diagonalizable. Thus, in order to produce inconsistent stability for the entire response near an unstable equilibrium, it is sufficient that all of the unstable de-coupled systems are individually stabilized. In this sense it is the most unstable eigenvalue of each equilibrium configuration (e.g., Fig. 7c that must be of concern. This concept will be further reinforced through a case study on an MDOF system, or better stated, an MDOF approximation of a distributed system. 

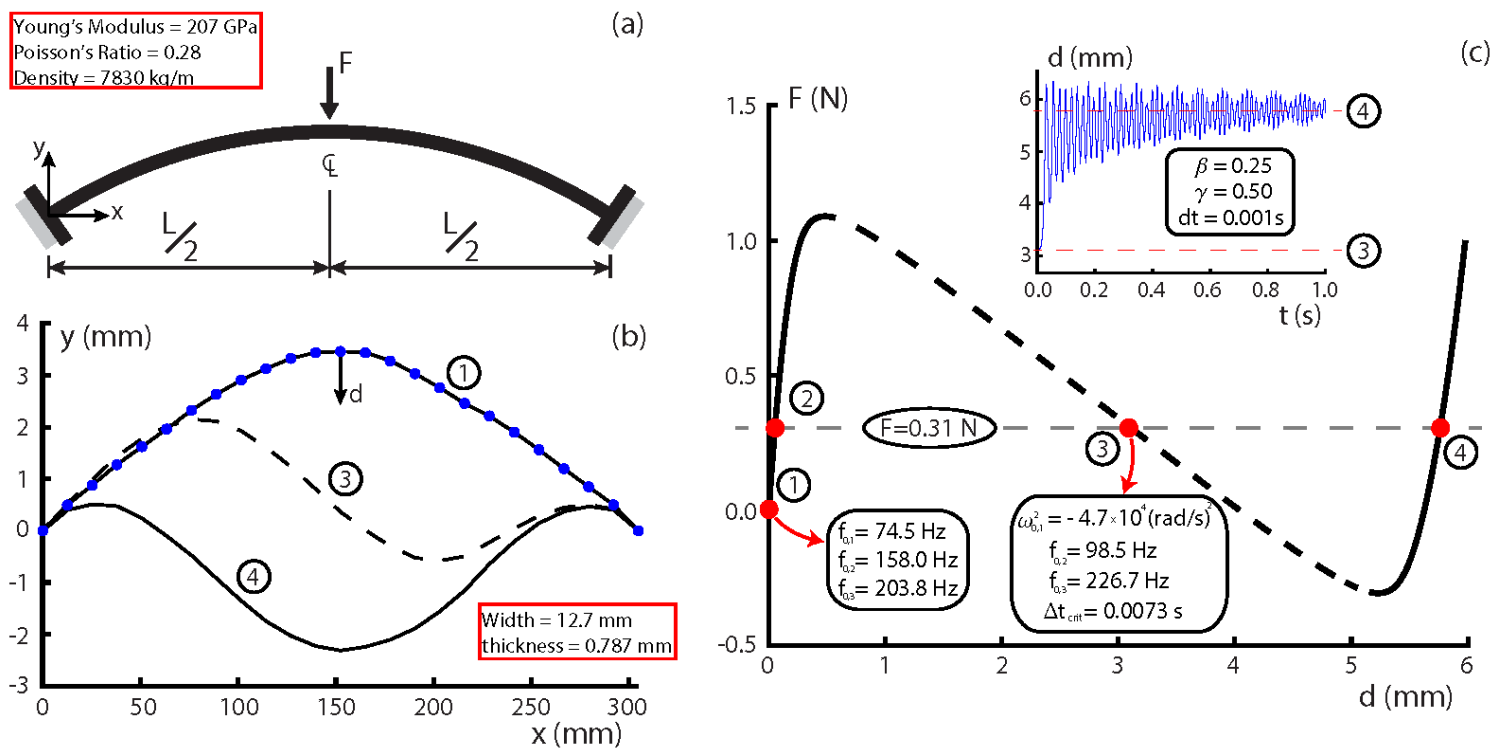

Fig. 10: A structural arch that exhibits multiple stable and unstable equilibria. (a) Schematic showing central point loading configuration and material properties. (b) Arch geometry; (solid curves) stable FEA equlibria, (dashed curve) unstable FEA equilibrium, and (blue circle) FEA nodal locations (shown on unloaded configuration). (c) Stable (solid) and unstable (dashed) static equilibrium relationship versus central displacement, $d$ (measured from unloaded configuration as shown), with specific loaded (points 2,3,4) and unloaded (point 1) arch configurations. An FE time series of the free decay from near the unstable equilibrium configuration is shown inset in part (c). Note that configuration 2 is omitted in part (b) as it is nearly indistinguishable from configuration 1.

\subsection{MDOF Arch}

Finally, we consider a case study on a distributed system that is modeled using finite element analysis (FEA). Fig. 10a shows the schematic of a curved beam under a point load at the midspan along with the beam material properties. Because curved beams are useful structural study cases and are the simplest case of multistable distributed structures, they are a good analogue of more complicated structures such as curved panels, and post-buckled plates. As this paper is not directed at modeling, we omit the details of the finite element formulation and instead focus on the behavior of the Newmark time discretization of the equation of motion.

Finite element discretization leads to the system of equations $M \ddot{x}+C \dot{x}+F_{\text {int }}(x)=F_{\text {ext }}$. Lumped mass, and mass proportional damping approximations were used to generate the matrices $M$ and $C$, while $F_{\text {int }}(x)$ is the (nonlinear) displacementdependent internal force, which is the primary output of the FE routine. This structure was based on an experiment that was performed in previous work; the interested reader is referred to $[9,11]$ for a more detailed discussion on the modeling of the beam. 

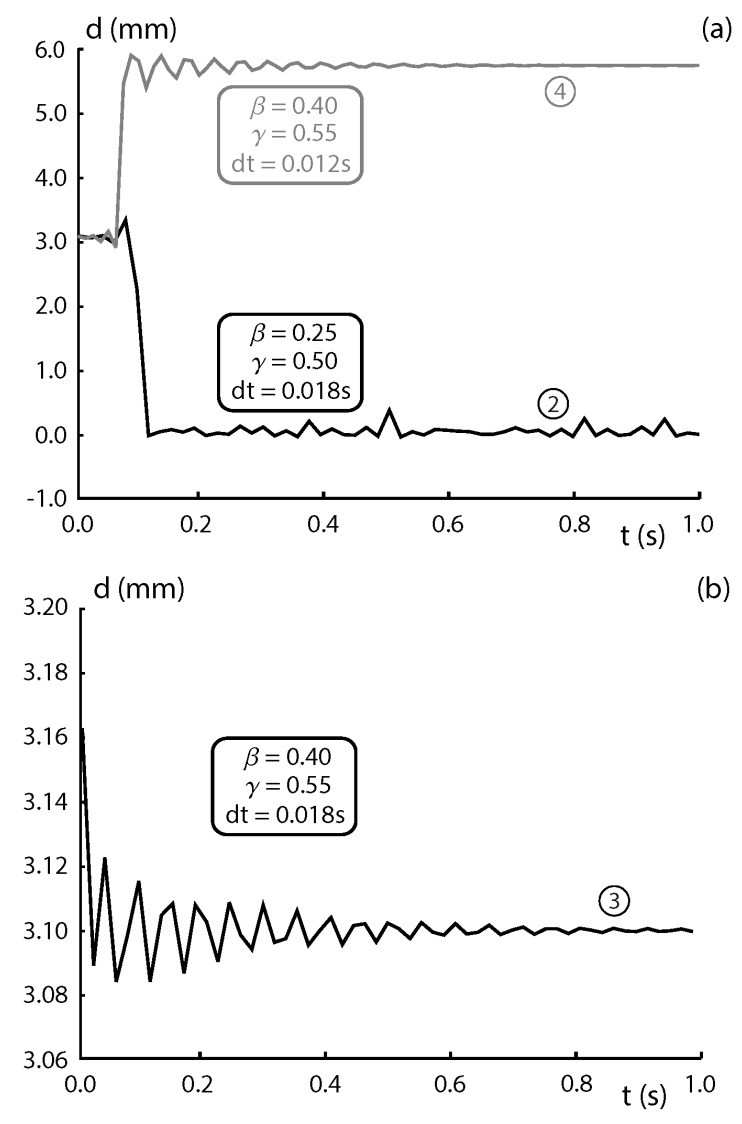

Fig. 11: Unforced dynamic response of arch obtained with the Newmark method using various time steps for an at-rest initial condition nearby but not on the unstable equilibrium configuration (point 3 in Fig. 10). (a) Two different Newmark parameter sets yielding consistent stability of configurations 2 and 4. (b) A Newmark parameter set yielding inconsistent stability, i.e., false stabilization of configuration 3. Note that the vertical axis is different for parts (a) and (b).

Fig. 10b shows the geometry of the unloaded configuration along with two of the static equilibrium configurations under a load of $F=0.31 \mathrm{~N}$. These equilibria are also shown in the force-displacement relationship in part (c). Two further unstable equilibria which are not shown may also exist, one nearly symmetric configuration, and one which is a near mirror image of configuration \#3. As it was based on an experiment, the beam includes geometric imperfections. The imperfection (note the visible asymmetry of the beam), induced a bias causing the system to prefer to pass through configuration \#3 rather than through a bifurcation in which configuration \#3, its mirror image, and a symmetric path all emerge (similar to the 2DOF system).

The static equilibrium relationship in part (c) shows only the midspan displacement and hence it is a projection. It is, however, the most informative in this case when combined with the full-field displacements shown in part (b). We will focus the discussion on the case $F=0.31 \mathrm{~N}$, which produces at minimum one unstable and two stable static equilibria (and potentially two more unstable unconnected branches [11] that cannot be obtained with a standard path following procedure). 
The unstable equilibrium configuration \#3, had only a single negative squared natural frequency. Also included in part (c) is a free decay time series (showing central displacement only) starting near the unstable equilibrium that gradually decays to configuration \#4. This could as easily have settled on configuration \#2, depending on which 'side' of configuration \#3 the ICs were chosen. This time series may be considered the 'truth' model, where the system damping alone is responsible for the decay.

Unlike the previous two case studies, this system has physical damping. The choice of mass proportional damping, however, yields a diagonalizable system. Mass proportional damping, and more generally Rayleigh damping (also leading to a diagonalizable system), are quite common in structural dynamics modeling, thus this assumption is not unreasonable. This fact again indicates that inconsistent stability might be a concern should the most unstable eigenvalue (in this case there is only one unstable eigenvalue for configuration \#3) be artificially stabilized. This is shown to be true in Fig. 11, where for $d t=0.012 \mathrm{~s}$, the response correctly decays to a stable configuration, while for $d t=0.018 \mathrm{~s}$ it incorrectly settles onto the unstable configuration \#3. As in the 2DOF case, these values are both well above the necessary condition for inconsistent stability value of $\Delta t=0.0073$, indicating that while this condition is necessary, it is not necessarily sufficient. This critical time step is also reasonably close to the $\Delta t=0.001 \mathrm{~s}$ used to generate the truth model.

The above behaviors are of particular concern in cases of disparate stability levels for different equilibria. In such a case, an analyst looking at the oscillations in the vicinity of a mildly stable (low stiffness) equilibrium may select a large time step, which will erroneously predict a stable response in the vicinity of a highly unstable (high negative stiffness) equilibrium. Furthermore, although it is a simple consequence of diagonalization, it is somewhat surprising to see that the stabilization of only a single modal direction (in a linearized system) may stabilize an unstable equilibrium.

\section{Conclusions}

In this paper we examine the connections between statics and dynamics, and the physical and numerical stability of conservative structures. It is shown that if the time step is selected to be too large, it is possible to erroneously stabilize unstable static equilibria, at least for small linearized oscillations in their vicinity. We develop a simple, and conservative,

expression given by $\Delta t<\sqrt{-1 / \beta \omega_{0}^{2}}$ (see Eq. (7)) for the most unstable static equilibrium in the domain of the response, that ensures consistent stability in the Newmark integrator. This condition is easy to check as it only requires information about the static equilibria.

In the examples discussed, is quite apparent that the chosen time step is too large for accurate results. However, the phenomenon examined here is not restricted to these particular systems or this specific time integrator. It is exhibited 
in almost all combinations of systems/discretizations (including for linear systems as demonstrated in [6]). Although the Newmark parameters that are used in most of this study are not standard, it is shown that even the standard parameters may exhibit temporary inconsistent stability. Furthermore, one could not rule out that nonlinearities might 'kick' a simulation with the standard parameters into the inconsistent stability region.

\section{Acknowledgements}

The authors acknowledge the support of Universal Technology Corporation (UTC) contract \# FA8650-10-D-3037, and High Performance Technologies, Inc. (HPTI) PETTT grant \# BY13-020SP.

\section{References}

[1] Hughes, T. J. R., 1987. The finite element method. Linear static and dynamic finite element analysis. Prentice-Hall International.

[2] Addison, P. S., Chan, A. H. C., Ervine, D. A., and Williams, K. J., 1992. “Observations on numerical method dependent solutions of a modified duffing oscillator". Communications in applied numerical methods, 8(8), pp. 519-528.

[3] Arnol'd, V. I., 1989. Mathematical Methods of Classical Mechanics, Vol. 60. Springer, NY.

[4] Thompson, J. M. T., and Hunt, G. W., 1973. A General Theory of Elastic Stability. J. Wiley London.

[5] Hughes, T. J. R., 1977. “A note on the stability of newmark's algorithm in nonlinear structural dynamics”. International Journal for Numerical Methods in Engineering, 11(2), pp. 383-386.

[6] Kalmar-Nagy, T., and Stanciulescu, I., 2014. “Can complex systems really be simulated?". Applied Mathematics and Computation, 227(C), pp. 199-211.

[7] Newmark, N. M., 1959. "A method of computation for structural dynamics”. In Proc. ASCE, Vol. 85, pp. 67-94.

[8] Thompson, J. M. T., and Stewart, H. B., 2002. Nonlinear dynamics and chaos. John Wiley \& Sons Inc.

[9] Wiebe, R., 2012. "Nonlinear dynamics of discrete and continuous mechanical systems with snap-through instabilities". $\mathrm{PhD}$ thesis, Duke University.

[10] Strogatz, S. H., 2001. Nonlinear Dynamics and Chaos: with Applications to Physics, Biology, Chemistry and Engineering. Perseus Books Group, USA.

[11] Chandra, Y., Wiebe, R., Stanciulescu, I., Virgin, L. N., Spottswood, S. M., and Eason, T. G., 2013. “Characterizing dynamic transitions associated with snap-through of clamped shallow arches”. Journal of Sound and Vibration, 332(22), pp. 5837-5855. 


\section{List of Figures}

1 Stability regions of the continuous (a) and discrete $($ b) systems . . . . . . . . . . . . . . . . .

2 Stability domain of discrete solution for unstable physical systems (a) $\omega_{0}^{2} \Delta t^{2} \rightarrow-\infty$; (b) $\gamma=0.5 \ldots \ldots$

3 A single-degree-of-freedom arch that exhibits multiple stable and unstable equilibria. (a) Schematic, (b) static equilibrium relationship, and (c) potential energy for $F=0$, and one example where $F \neq 0$. . . .

4 Unforced response of the SDOF system obtained using the Newmark method $(\beta=0.4$ and $\gamma=0.55)$ for various time steps $(d t=0.01 \mathrm{~s}$ and $d t=0.10 \mathrm{~s}$ initiated from opposite sides for clarity). (a) Time series showing inconsistent stability for $d t=1.0 \mathrm{~s}$. (b) Basins of attraction for consistent stability (unshaded), inconsistent stability (dark gray), and non-converging (light gray) solutions for $d t=1.0 \mathrm{~s} . F=0.0, x(0)=$ $\pm 0.1, \dot{x}(0)=0.0 \ldots \ldots \ldots \ldots \ldots \ldots \ldots$

5 Forced response of the SDOF system obtained using the Newmark method $(\beta=0.4$ and $\gamma=0.55)$ for various time steps (black) with overlayed instantaneous stable (red/dark shaded curve) and unstable (gray/light shaded curve) static equilibria. $F=0.1 \sin \left(\frac{2 \pi}{10} t\right), x(0)=0.01, \dot{x}(0)=0.0 \ldots \ldots \ldots$

6 Unforced response of the SDOF system, now for $k / m=2800$, obtained using the Newmark method with standard parameters $(\beta=0.25$ and $\gamma=0.5)$ for $d t=1 \mathrm{~s}, x(0)=0.1, \dot{x}(0)=0.0 \ldots \ldots$

7 A two-degree-of-freedom arch that exhibits multiple stable and unstable equilibria. (a) Schematic, (b) static equilibrium relationship, and (c) stability via natural frequency (squared) of the five equilibria (red empty circles) present under $F=0.05 . \ldots \ldots \ldots \ldots \ldots \ldots$

8 True response of the 2DOF system under constant force $F=0.05$ obtained using the Newmark method ( $\beta=0.4$ and $\gamma=0.55)$ for $d t=0.1 \mathrm{~s}$. (a) Phase portrait in $\left(\theta_{1}, \theta_{2}\right)$ projection, i.e., angular velocities are not shown. (b) Time series of $\theta_{1}$. The circles denote the positions of the static equilibria. . . . . . . . .

9 Consistent and inconsistent stability of the 2DOF system under dead load $F=0.05$ obtained using the Newmark method ( $\beta=0.4$ and $\gamma=0.55$ ) for various time steps. (a) Consistent stability for $d t=6 \mathrm{~s}$. (b) Inconsistent stability for $d t=8 \mathrm{~s}$. All responses initiated near unstable equilibria. . . . . . . . . . . 
10 A structural arch that exhibits multiple stable and unstable equilibria. (a) Schematic showing central point loading configuration and material properties. (b) Arch geometry; (solid curves) stable FEA equlibria, (dashed curve) unstable FEA equilibrium, and (blue circle) FEA nodal locations (shown on unloaded configuration). (c) Stable (solid) and unstable (dashed) static equilibrium relationship versus central displacement, $d$ (measured from unloaded configuration as shown), with specific loaded (points 2,3,4) and unloaded (point 1) arch configurations. An FE time series of the free decay from near the unstable equilibrium configuration is shown inset in part (c). Note that configuration 2 is omitted in part (b) as it is nearly indistinguishable from configuration $1 \ldots \ldots \ldots \ldots \ldots \ldots \ldots$

11 Unforced dynamic response of arch obtained with the Newmark method using various time steps for an atrest initial condition nearby but not on the unstable equilibrium configuration (point 3 in Fig. 10). (a) Two different Newmark parameter sets yielding consistent stability of configurations 2 and 4. (b) A Newmark parameter set yielding inconsistent stability, i.e., false stabilization of configuration 3 . Note that the vertical

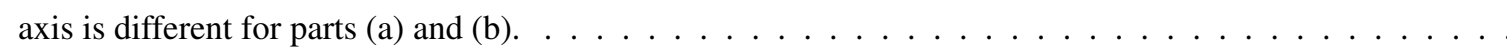

